\title{
MITTEILUNGEN
}

\section{Parlamentsarmee zwischen Grundgesetz und Völkerrecht. Eine Veranstaltung der DVParl am 24. März 2010 in Berlin}

„Die Sicherheit der Bundesrepublik wird auch am Hindukusch verteidigt. “1 Mit dieser Äußerung machte Verteidigungsminister Peter Struck 2002 deutlich, dass für die Bundeswehr, die klassische Funktion der Landesverteidigung nicht mehr an erster Stelle steht. In der öffentlichen Debatte macht sie vielmehr durch Entwicklungen in den Auslandseinsätzen auf sich aufmerksam. Dabei richtet sich das Augenmerk auch auf die rechtliche Grundlage der Einsätze. Allein die unterschiedlichen Bezeichnungen des militärischen Einsatzes in Afghanistan zeigen, wie zwiespältig die Situation ist. Von „Stabilisierungseinsatz“ bis hin zu „kriegsähnlichen Zuständen“" wurden viele Termini verwendet, um die Situation begrifflich zu fassen. Dabei kommt es nicht so sehr auf die Benennung, sondern vielmehr auf die verfassungsrechtlichen Folgen, die die unterschiedlichen Definitionen mit sich bringen, denn ob ein Einsatz zur Friedenssicherung oder ein Krieg im Sinne des Völkerrechts vorliegt, bestimmt die Rechtslage für die Ausführung militärischer Einsätze maßgeblich.

Die Unklarheiten in der Rechtslage sowie die verschiedenen Auffassungen zu verfassungsmäßigen Regelungen der Auslandseinsätze thematisierte die Deutsche Vereinigung für Parlamentsfragen in einer Veranstaltung am 24. März 2010, zu der die Völkerrechtler Georg Nolte (Humboldt-Universität zu Berlin) und Andreas von Arnauld (Universität der Bundeswehr in Hamburg) eingeladen waren. Die Leitung übernahm Georg Paul Hefty (Redakteur der Frankfurter Allgemeinen Zeitung).

Mit zwei zentralen Fragen leitete er die Diskussion ein: Die erste bezog sich auf den rechtlichen Konflikt, in der sich deutsche Soldaten befinden, wenn sie innerhalb der Verbände der „Gastnationen“ nach deren Rechtsauffassungen mitkämpfen. Die zweite richtete sich auf die strafrechtliche Verantwortung, die durch die Situation entsteht, dass sich Truppen anderer Nationen unter deutschem Kommando nach eigenem Verständnis der Kriegsführung in militärische Auseinandersetzungen begeben.

Dazu führte Georg Nolte drei zentrale Thesen an: Erstens stehen die grundlegenden Regeln des Grundgesetzes und des Völkerrechts im Hinblick auf Bundeswehreinsätze nicht im Widerspruch zueinander; zweitens soll das Grundgesetz bei Fragen der Führung bewaffneter Konflikte völkerrechtskonform ausgelegt werden, und drittens hat das Parlament für die völkerrechtskonforme und verfassungsgemäße Durchführung der Bundeswehreinsätze keine größere Verantwortung als die Regierung. Vielmehr nehme das Parlament hinsichtlich des Schutzes der Grund- und Menschenrechte eine hervorgehobene Stellung ein.

Die erste These unterstützte Nolte durch Rekurs auf eine Reihe völkerrechtlicher Regelungen, die das Grundgesetz aufgenommen und für die Bundesrepublik Deutschland bindend ausgelegt hat. Zwar besteht danach ein Spannungsverhältnis zwischen Grundgesetz und Völ-

1 Peter Struck in einer Pressekonferenz am 5. Dezember 2002, zitiert nach Severin Weiland, Struck bastelt an eigenen Schulterklappen, Spiegel online vom 5. Dezember 2002, http://www.spiegel. de/politik/deutschland/0,1518,225810,00.html (Abruf am 25. August 2010).

Zeitschrift für Parlamentsfragen (ZParl), Heft 3/2010, S. 709 - 715 
kerrecht, jedoch kann von einem Widerspruch nicht die Rede sein - schon deshalb nicht, weil das Völkerrecht die Staaten weder zur Teilnahme an militärischen Missionen verpflichtet, noch bestimmte Methoden der Kampfführung vorschreibt. Nolte sieht den Kern des Spannungsverhältnisses zwischen Grundgesetz und Völkerrecht in der unterschiedlichen Auffassung der Menschenwürde. Das Bundesverfassungsgericht hat in seinem Urteil zum Luftsicherheitsgesetz festgestellt, dass die bewusste Tötung unbeteiligter Zivilisten in bewaffneten Konflikten auch als verhältnismäßige Nebenfolge bei der Bekämpfung eines legitimen militärischen Ziels verfassungsrechtlich nicht zulässig ist. Die Begründung fußt auf der Unvereinbarkeit ziviler Opfer mit dem Recht auf Leben (Art. 2 Abs. 2 Satz 1 GG) und der Menschenwürdegarantie (Art. 1 Abs. 1 GG). Das humanitäre Völkerrecht hingegen sieht die bewusste Tötung unbeteiligter Zivilisten in bewaffneten Konflikten durchaus als „legitimen zivilen Begleitschaden“. Nolte empfiehlt dem Bundesverfassungsgericht, die Menschenwürde zu konkretisieren und legt nahe, dem Muster aus dem Völkerrecht zu folgen. Dessen Konkretisierung ist ein Resultat aus vielen unterschiedlichen, auch modernen Konflikten und daher angemessen, um situationsgerecht auf Gefahren bei Militäreinsätzen im Rahmen asymmetrischer Konflikte zu reagieren. Nolte untermauerte seine zweite These damit, dass die Regeln des humanitären Völkerrechts aufgrund der universellen Akzeptanz eine hohe Legitimität besitzen, und er betonte, dass der richtige Ort für eine Einschränkung der völkerrechtlichen Regelungen von Auslandseinsätzen nicht die Verfassung sei. Falls der Bundestag Bundeswehreinsätze vorsieht, hat er im Rahmen seiner weitreichenden Kontrollkompetenzen die Möglichkeit, diese anhand eines allgemeinen Gesetzes strengeren Maßstäben, als sie das Völkerrecht vorsieht, auszusetzen und seiner herausragenden Verantwortung für den Grund- und Menschenrechtsschutz nachzukommen.

Zusammenfassend verdeutlichte Nolte noch einmal, dass kein Widerspruch zwischen Grundgesetz und humanitärem Völkerrecht besteht. Spannungsverhältnisse, die die Soldaten im Rahmen von Auslandseinsätzen in rechtliche Konflikte bringen, sind durch eine völkerrechtskonforme Auslegung des Grundgesetzes zu beheben. Des Weiteren sprach sich Nolte dafür aus, weitreichende Regulierungen von Kampfhandlungen in bestimmten Einsätzen der Bundeswehr, die über das Völkerrecht hinausgehen, anhand von allgemeinen Gesetzen und nicht auf Verfassungsebene vorzunehmen.

Andreas von Arnauld bekundete in seinem Vortrag inhaltliche Übereinstimmungen zu Nolte und konzentrierte sich auf die folgenden Fragen: Unterliegen deutsche Soldaten im Auslandseinsatz weitreichenderen Beschränkungen als Soldaten aus anderen Staaten? Und an welchen Regeln sollen sie sich im Ausland orientieren?

Da sich nicht alle Länder an jedem völkerrechtlichen Übereinkommen beteiligt haben, gibt es zwar Unterschiede in den Maßstäben und Beschränkungen der Kampfhandlungen. Viel ausschlaggebender wirkt sich jedoch die Tatsache aus, dass die Nationalstaaten die gemeinsamen völkerrechtlichen Einschränkungen unterschiedlich interpretieren. Es kann jedoch nicht von einem deutschen Sonderweg ausgegangen werden, da die völkerrechtlichen Regelungen im Allgemeinen kollektiv ratifiziert wurden. Die weitreichenden Beschränkungen deutscher Soldaten im Auslandseinsatz sind nur in geringem Maße auf verfassungsrechtliche beziehungsweise völkerrechtliche Regelungen zurückzuführen. Vielmehr ergeben sich Restriktionen insbesondere aus dem jeweiligen Mandat, das der Bundestag der Bundeswehr für den Einsatz erteilt hat.

Mit Blick auf die Regeln, an denen sich die Soldaten im Auslandseinsatz orientieren sollen, präzisierte Arnauld die prekäre juristische Situation wie folgt: Kern des Problems sei 
die Tatsache, dass Auslandseinsätze der Bundeswehr im Rahmen des Grundgesetzes über den Verteidigungsfall des Art. 87a Abs. 2 GG hinaus nicht dezidiert vorgesehen seien, sich vielmehr über Artikel 24 Abs. 2 GG in Form einer Nebenverfassung entwickelt hätten, wodurch Interpretations- und Handlungsfreiräume entstünden, die zu einem Spannungsverhältnis zwischen Grundgesetz und Völkerrecht führen könnten.

Am Beispiel der Bekämpfung der Piraterie am Horn von Afrika wird deutlich, dass es sich empfiehlt, die internationale militärische Kooperation um eine justizielle Komponente zu erweitern: Während das Grundgesetz die Vorführung Festgenommener vor einen Richter binnen 48 Stunden vorschreibt, hat der Europäische Gerichtshof für Menschenrechte eine Frist von 14 Tagen als ausreichend anerkannt. Das Völkerrecht hingegen sieht eine sofortige Vorführung vor.

Des Weiteren sprach sich Arnauld dafür aus, die Schranken der Auslandseinsätze weder zu niedrig noch zu hoch zu setzen, um auf unvorhersehbare Situationen auf unbekanntem Terrain angemessen reagieren zu können. Dabei bewährt sich der Grundsatz der Verhältnismäßigkeit als eine offene Struktur im Grund- und Menschenrechtsschutz. Arnauld befürwortete hier eine Auslegung des Grundgesetzes im Sinne der völkerrechtlichen Vorgaben, da diese eine solide Grundlage darstellen, um in verschiedenen Einsätzen verschiedenen Verhältnissen und Bedingungen gerecht zu werden. Kein Auslandseinsatzgesetz, sondern vielmehr einsatzspezifische Regeln für Beobachtungs- beziehungsweise Kampfeinsätze oder Einsätze zu Land oder zu Wasser sind in der aktuellen Situation das hilfreiche Mittel.

In Bezug auf Auslandseinsätze der Bundeswehr sah Arnauld die Forderungen des Grundgesetzes im Wesentlichen mit denen des Völkerrechts vereinbar. Er plädierte jedoch dafür, juristisch klarere Regelungen zu schaffen und das Grundgesetz auf Situationen zu befragen, die im Auslandseinsatz entstehen könnten. Weiterhin machte er deutlich, dass er die Maßstäbe des Völkerrechts für angemessen erachtet.

Im Mittelpunkt der abschließenden Diskussion stand die von Hans-Peter-Bartels (MdB, SPD-Fraktion) aufgeworfene Frage, inwieweit das Grundgesetz geändert werden müsse, um Rechtsklarheit für Auslandseinsätze zu erreichen. Nolte betonte erneut, dass das Grundgesetz solche Einsätze nicht vorsieht, jedoch auch nicht der angemessene Ort für ihre Regelung sei. Arnauld hingegen differenzierte in ein Recht zum Auslandseinsatz und ein Recht im Auslandseinsatz. Für ersteres empfahl er eine klarere Regelung, die vom Bundesverfassungsgericht in Zusammenarbeit mit dem Gesetzgeber erarbeitet und in das Grundgesetz aufgenommen werden solle. Bezüglich der Rechtslage im Ausland machte Arnauld auf die Grundrechte im Grundgesetz aufmerksam und forderte auf, sich an diesen zu orientieren. Anstatt das Grundgesetz in dieser Hinsicht zu verändern oder Regelungen auf verfassungsrechtlicher Ebene zu schaffen, solle lieber das Grundgesetz befragt werden.

Insgesamt hat die Abendveranstaltung dank der ausführlich und kontrovers geführten Diskussion ein differenziertes Bild geliefert. Einerseits waren sich die Referenten darüber einig, dass das Grundgesetz im Sinne der Rechtsklarheit möglichst völkerrechtskonform ausgelegt werden soll; andererseits hatten sie abweichende Vorstellungen darüber, auf welcher Ebene diese Auslegung beziehungsweise Regelung stattzufinden hat. Unmissverständlich klar wurde auch, dass das Grundgesetz und das Völkerrecht keinesfalls als konkurrierende Rechtskataloge zu betrachten sind. Beide entfalten für Bundeswehreinsätze ihre Geltung. Die juristische Feinabstimmung zwischen dem Grundgesetz und dem Völkerrecht ist jedoch noch ergänzungsbedürftig.

Servan Deniz 